\title{
Increased incidences of post-anesthesia care unit overstay and postoperative subcutaneous emphysema in transoral vestibular endoscopic thyroidectomy: an audit report
}

\section{Lan, Kuo-Mao ${ }^{1,3}$, Wang, Kuei-Fen ${ }^{1}$, Lin, Yao-Tsung ${ }^{2,}{ }^{3}$, Wang Li-Kai ${ }^{1}$, Wu, Su-Zen ${ }^{1}$,} Chen, Jen-Yin ${ }^{1,4}$

1. Dept. of Anaesthesiology, Chi Mei Medical Center, Tainan, Taiwan

2. Dept. of Anaesthesiology, Chi Mei Medical Center, Liouying, Tainan, Taiwan

3. Dept. of Food Science and Applied Biotechnology, National Chung Hsing University, Taichung, Taiwan

4. Dept. of the Senior Citizen Service Management, Chia Nan University of Pharmacy and Science,

Tainan, Taiwan

\section{Background and Goal of Study}

Transoral vestibular endoscopic thyroidectomy has been developed for a better cosmetic result. In our hospital, the new surgical technique has been introduced since 2015. Quality control report revealed increased PACU overstay incidence. Gas insufflations into neck region also raised concern. Herein we present a retrospective audit.

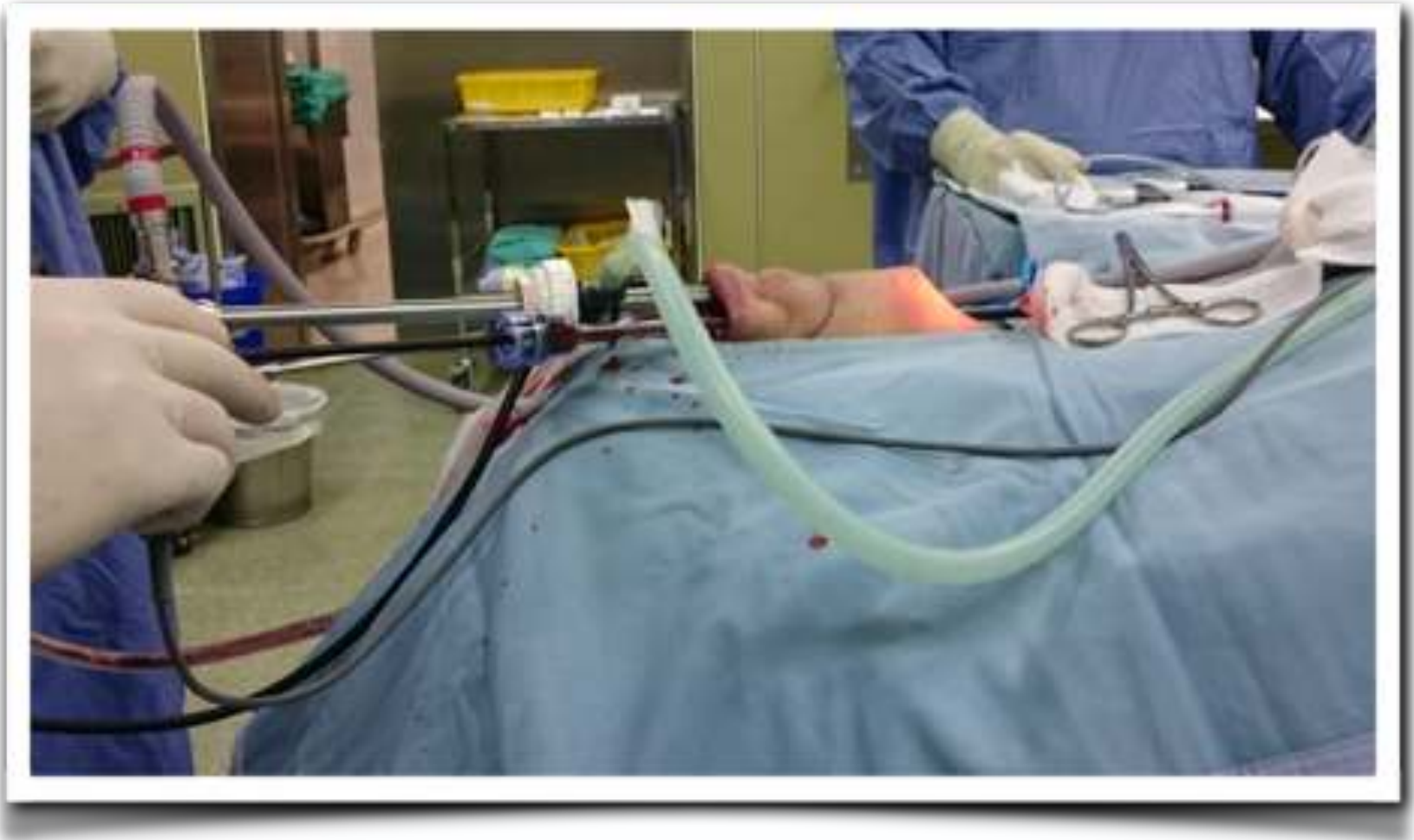

\section{Materials and Methods}

A total of 80 patients received TVET from Oct. 2015 to Sep. 2016. General anesthesia was induced and maintained with sevoflurane/desflurane in oxygen/air mixture (FiO2 0.4-0.5; 1-2 L/min). Under nasotracheal intubation and neuromonitoring, all operations were performed by one experienced laparoscopic surgeon. He used laparoscopic instruments to establish 3 subcutaneous tunnels from oral vestibule to front neck and create an air pocket with $6 \mathrm{mmHg} \mathrm{CO} 2$ insufflation. During PACU care after surgery, nurses checked patient's neck, shoulder and chest at 15 minutes if subcutaneous emphysema develops. PACU staying time and adverse events were recorded.

\section{Results}

The incidence $(7 / 80=8.8 \%)$ of PACU overstay (longer than 2 hours) was higher than average monthly incidence (3.4\%) ranging from $2.8 \%$ to $3.9 \%$. SE was found in 31 patients $(31 / 80=38.8 \%)$. Three of them had stridor $(3 / 31=9.7 \%)$ and ten exhibited temporary difficulties of breath or swallow $(10 / 31=32.3 \%)$. After oxygen therapy, none experienced desaturation (SPO2 < 90\%). Nonetheless, none stayed in PACU longer than 3 hours.

\section{Discussion}

PACU overstay may result in operating room congestion, reduced patient satisfaction and increased financial burden. In our audit, SE occurred in more than one third patients and the incidence of PACU overstay was increased. PACU overstay was related to SE and its associated sequelae which was caused by $\mathrm{CO} 2$ insufflation near airway during TVET procedures. SE occurs as a benign course and resolves spontaneously in most cases. SE is easily overlooked when this minor complication goes without other complications. It may go unrecognized because of rapid absorption of $\mathrm{CO} 2$. However, it may progress to pneumothorax or mediastinal emphysema in rare conditions. Hence, $\mathrm{SE}$ is a concern for anesthesiologists because of potential risks of subsequent major sequelae.

\section{Conclusion}

TVET is a new technique, however, it is not completely proven to be safe and without potential morbidity. Increased incidences of PACU overstay and SE after TVET were noted. Further investigation is warranted. 